\title{
ACE2 mutation might explain lower COVID-19 burden in malaria endemic areas
}

\author{
Auley $\mathrm{De}^{1} \cdot$ Aparna Tiwari $^{1,2} \cdot$ Manoswini Dash $^{1,2} \cdot$ Abhinav Sinha $^{1}[$ \\ Received: 5 January 2021 / Accepted: 11 January 2021 / Published online: 25 January 2021 \\ (c) Japan Human Cell Society 2021
}

Keywords Malaria $\cdot$ COVID-19 $\cdot$ ACE2 variants $\cdot$ Natural selection

The evolving micro-epidemiology of COVID-19 has raised several intriguing concerns including its disproportionately lower burden in malaria-endemic regions. Although there are various explanations cited for this, evolutionary genetic linkages through Angiotensin-Converting Enzyme 2(ACE2) polymorphisms have been superficially mentioned but not scrutinized further [1].

ACE2 regulates blood pressure through Angiotensin II and Angiotensin (1-7), but also acts as an entry receptor for the SARS-CoV-2 through its spike glycoproteins. The pathogenesis of COVID-19 is said to depend on the relative interplay between different ACE2 elevating and lowering factors (Fig. 1).

A number of ACE2 polymorphisms are known to be associated with the pathogenesis of a variety of non-communicable diseases such as cardiovascular disorders (including hypertension) and diabetes mellitus [2]. As evident, none of ACE2-associated diseases are communicable except malaria, whose pathogenesis (severe malaria) is strongly hypothesised to be protected by a certain ACE2 SNP [3, 4]. It is worthwhile to note that out of 25 different ACE2

Auley De, Aparna Tiwari and Manoswini Dash have contributed equally.

Abhinav Sinha

abhinav@mrcindia.org

Auley De

de.auley@gmail.com

Aparna Tiwari

aparna@nimr.org.in

Manoswini Dash

manoswini.dash@gmail.com

1 ICMR-National Institute of Malaria Research, New Delhi 110077, India

2 Kumaun University, Nainital 263001, Uttarakhand, India
SNPs studied for cardiovascular and related diseases in different ethnicities with a minor allele frequency of $>1 \%$, only one ACE2 SNP (T-allele of rs2106809) has been shown to be negatively associated with severe malaria [4] and positively associated with hypertension through lowered plasma Ang (1-7) level. As Ang (1-7) is mostly derived from Ang II through ACE2, collectively, this indirectly implies that the $\mathrm{T}$-allele of rs 2106809 down-regulates the expression of ACE2 [5, 6]. As per the malaria-hypertension hypothesis, the T-allele of rs 2106809 might be positively selected in populations that are chronically exposed to malaria and hence increase the likelihood of Ang II-mediated hypertension via downregulating ACE2 [7]. Besides, there are other rs2106809-tagged SNPs, that are under strong linkage disequilibrium with rs2106809 and could have functional significance [5]. Interestingly, ACE2 rs2285666 is one such tagged-SNP that is found to be associated with lower plasma ACE2 level [5].

We, therefore, rationally speculate that the malariaexposed populations may have a preponderance of compensatory genetic variants (T-allele of rs2106809 and its tagged-SNPs), that protect from severe malaria in childhood, but eventually aggravate the risk of hypertension in adulthood through increased circulating Ang II level [3, 4]. This epidemiological interplay is thought to become even more complex during exposure to the SARS-CoV-2, wherein ACE2 downregulation tends to protect such population from SARS-CoV-2 infection (decreased prevalence of infection), but compensatory Ang II upregulation tends to increase the severity in the infected population (increased proportions of severe infection). The net effect may be compounded/ masked in the presence of other ACE2-upregulating conditions such as smoking, higher age, and comorbidities, thus leading to a mixed epidemiological and clinical profile, as is currently apparent. Thus, the epidemiology and pathogenesis of COVID-19 in populations living in malaria-endemic 


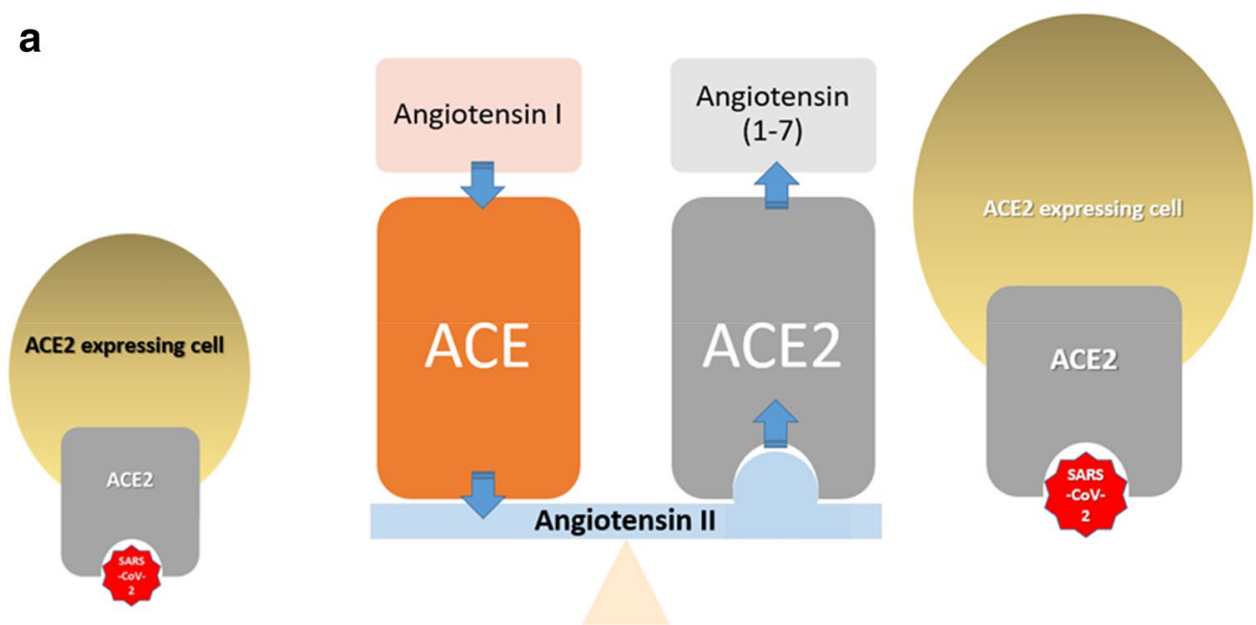

b

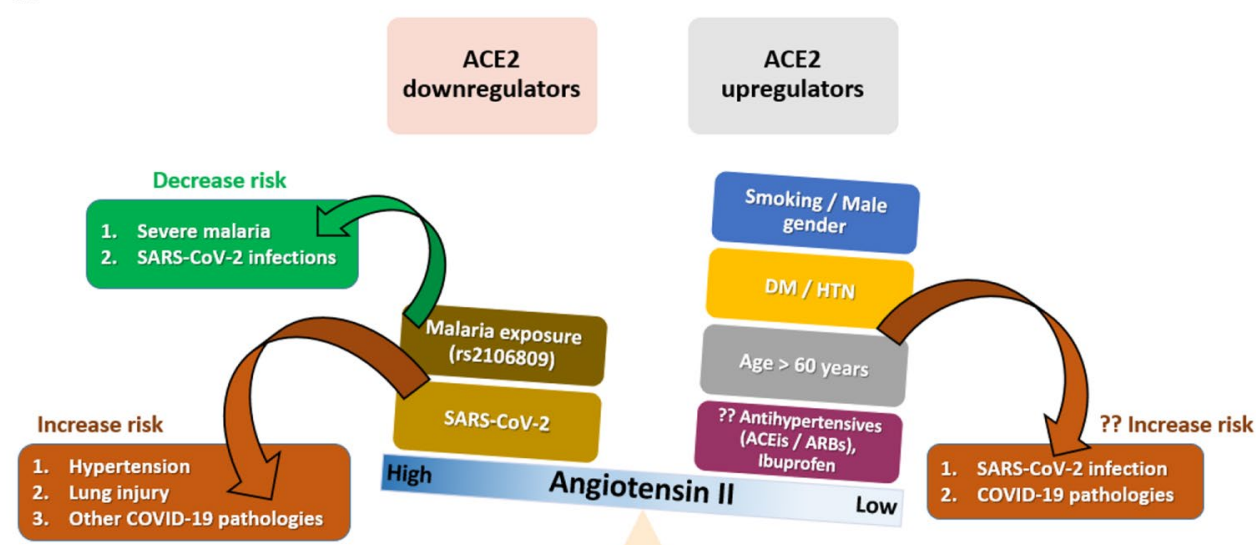

Fig. 1 Ultra-simplified cartoon of the Renin-Angiotensin System (RAS) with the relevant components (top panel): The system balances the levels of Angiotensin II (light blue bar) through relative concentrations of Angiotensin-Converting Enzyme (ACE; orange rectangle) which converts Angiotensin I to Angiotensin II and Angiotensin-Converting Enzyme 2 (ACE2; grey rectangle) which converts Angiotensin II to Angiotensin (1-7). The catalytic site of ACE2 is shown to bind Angiotensin II. The SARS-CoV-2 (red spiked solid circle) is shown to use the same catalytic site of ACE2 for its entry into an ACE2 expressing cell (yellow shaded oval). Various relevant factors that disturb the crucial Angiotensin II balance is shown (bottom panel). Angiotensin II levels are increased by ACE2 downregulators

countries is hypothesised to be dependent on the relative interplay of the specific host genetic epidemiology and other related factors (Fig. 2).

As per our best knowledge, no published study has analysed the aforesaid association apart from the two indirect published evidence. A case-control study that investigated the association of rs2106809-tag SNP rs2285666 (and different exonic ACE2 variants) with COVID-19 (and its severity) could not find a statistically significant association possibly due to factors related to the study design and sample size [8]. Another study that supports our hypothesis was done
(ACE2 rs2106809) and SARS-CoV-2 infection itself. Resulting low ACE2 offers protection from severe malaria (through high Angiotensin II) and from SARS-CoV-2 infection (through decreased ACE2 receptors). Concurrently, ACE2 downregulators tend to increase the risk of hypertension and COVID-19 pathologies due to increased Angiotensin II. On the other hand, ACE2 upregulators may increase the risk of SARS-CoV-2 infection (through increased receptors) and related pathology (through high viral load due to increased receptors). The final COVID-19 pathogenesis depends on the relative balance between ACE2 down- and up-regulators (DM Diabetes Mellitus; HTN Hypertension)

on Chinese population that tested rs2106809 (among others including rs2285666) for increased SARS susceptibility and worse outcomes suggested a protective effect of rs 2106809 T-allele on SARS susceptibility ( $O R=0.9$; statistically insignificant) [9]. However, it has to be noted that rs2106809 may not act independently as a genetic determinant of SARSCoV-2 infection/severity [10].

A brief analysis of the available data on the distribution of COVID-19 prevalence (cases per million population) and severity (in terms of case fatality rate per $1000 \mathrm{popu}-$ lation) in malaria endemic countries with information on 


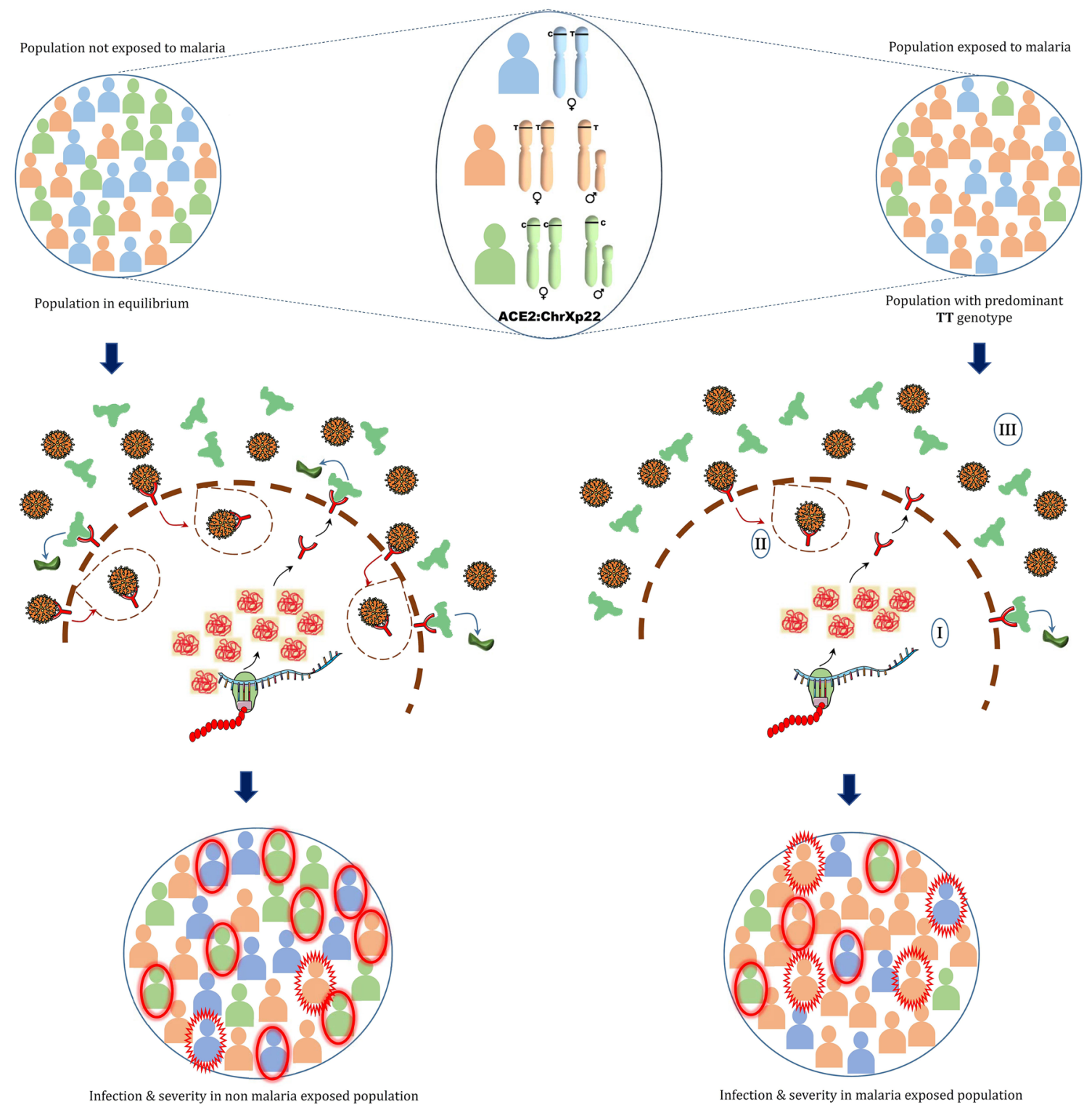

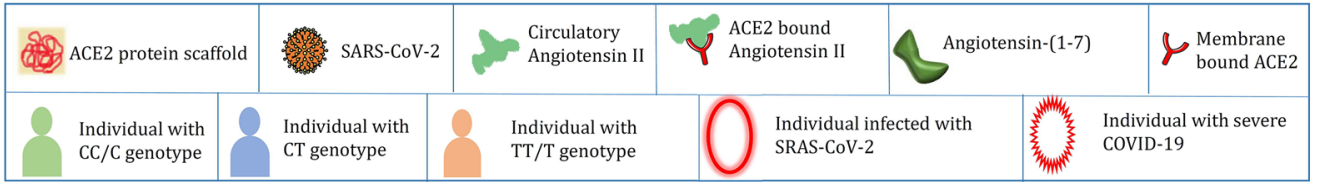

Fig. 2 Proposed hypothesis explains that the malaria exposed population (right) has a relatively higher prevalence of naturally selected ACE2 rs2106806 TT/T genotype (pink) as compared to the population not exposed to malaria (left). The $\mathrm{T}$ allele is known to be associated with decreased ACE2 expression and hence lesser receptors for SARS-CoV-2, therefore, reducing the chances of viral entry into the cells. The reduced ACE2 also restricts the conversion of Ang II

the population having the T-allele of rs 2106809 currently does not support the proposed hypothesis (data not shown here) due to many factors. Principal among them include to Ang (1-7), thereby elevating the level of circulating Ang II which precipitates COVID-19 severity due to lung injury and other negative effects via the Angiotensin Type 1 Receptor $\left(\mathrm{AT}_{1} \mathrm{R}\right)$. The pathogenesis of COVID-19 thus differs in the two populations: lesser prevalence of SARS-CoV-2 infections but a higher proportion of severe COVID-19 in malaria exposed population as compared to population not exposed to malaria

those related to ACE2 T-allele (available data lacks internal/ external validities, lack of individual-centric data on both ACE2 SNP and COVID-19), malaria (endemicity is highly 
variable even within an "endemic" country/state), COVID19 (severity outcome is not available, evolving nature of the pandemic) and health systems (variations in case definitions, diagnostic methods, surveillance strategies). However, if the available data on COVID-19 prevalence and mortality be standardized for some or more of the non-genetic determinants, the role of rs 2106809 could be better evaluated. In addition, the attributable fractions of each of these genetic and non-genetic determinants are not currently available which makes it difficult to add a corresponding weightage to each of them. This becomes more valid if the population under consideration has more than two determinants, so that the weighted protective effect of one may be compromised by the weighted aggravating effect/s of other/s and vice versa

These factors preclude powerful analyses of the available data needed for validating the proposed hypothesis and hence reinforce the need to perform powerful and targeted studies with specific objectives. Amidst all possible uncertainties in the proposed conjecture, the hypothesis certainly warrants confirmation in the immediate future as a testable body of science. We propose that the hypothesis linking malaria-hypertension-COVID-19 stands logical enough to be best tested through powerful case-control studies in the population that has developed a COVID-19-related outcome and evaluate the association with their $A C E 2$ variants. Simultaneously, the mechanism through which the intronic mutations (rs2106809 and rs2285666) affect the expression and regulation of ACE2 needs to be elucidated. It would be fascinating to study the ACE2 expression profile in differentially affected COVID-19 and non-affected population, particularly under different malaria endemicity zones, under different genetic (rs2106809 and tag-SNPs) and non-genetic non-modifiable (age and gender) and modifiable (e.g., smoking) conditions and also in presence of comorbidities and the treatment thereof.

Acknowledgements We would like to acknowledge the Director, ICMR-National Institute of Malaria Research for encouragement and support for preparing the manuscript. AD \& MD were supported by the Indian Council of Medical Research, New Delhi and AT by Council of Scientific \& Industrial Research, New Delhi.

Author contributions $\mathrm{AD}, \mathrm{AT}$ and $\mathrm{MD}$ contributed equally in literature search, data acquisition, analyses and interpretation, drafting the manuscript and revising it critically. $\mathrm{AD}, \mathrm{MD}$ and $\mathrm{AS}$ generated figures. $\mathrm{AD}$ and AS conceptualized the work. AS analyzed and interpreted the data, critically revised and edited the manuscript. All the authors finally approved the version of the manuscript to be published.

\section{Compliance with ethical standards}

Conflict of interest The authors declare that they have no conflict of interest.

\section{References}

1. Napoli PE, Nioi M. Global spread of coronavirus disease 2019 and malaria: an epidemiological paradox in the early stage of a pandemic. J Clin Med. 2020;9(4):1138.

2. Bosso M, Thanaraj TA, Abu-Farha M, Alanbaei M, Abubaker J, Al-Mulla F. The two faces of ACE2: the role of ACE2 receptor and its polymorphisms in hypertension and COVID-19. MolTher Methods Clin Dev [Internet]. 2020;18(September):321-7. https:// doi.org/10.1016/j.omtm.2020.06.017.

3. Gallego-Delgado J, Rodriguez A. Malaria and hypertension. Another co-evolutionary adaptation? Front Cell Infect Microbiol. 2014;4:121.

4. Dhangadamajhi G, Mohapatra BN, Kar SK, Ranjit M. Gene polymorphisms in angiotensin I converting enzyme (ACE I/D) and angiotensin II converting enzyme $(\mathrm{ACE} 2 \mathrm{C} \rightarrow \mathrm{T}$ ) protect against cerebral malaria in Indian adults. Infect Genet Evol. 2010;10(2):337-41.

5. Chen YY, Zhang P, Zhou XM, Liu D, Zhong JC, Zhang CJ, et al. Relationship between genetic variants of ACE2 gene and circulating levels of ACE2 and its metabolites. J Clin Pharm Ther. 2018;43(2):189-95.

6. Liu D, Chen Y, Zhang P, Zhong J, Jin L, Zhang C, et al. Association between circulating levels of ACE2-Ang-(1-7)-MAS axis and ACE2 gene polymorphisms in hypertensive patients. Med (United States). 2016;95(24):1-6.

7. Gallego-Delgado J, Walther T, Rodriguez A. The high blood pressure-malaria protection hypothesis. Circ Res. 2016;119(10):1071-5.

8. Gómez J, Albaiceta GM, García-Clemente M, López-Larrea C, Amado-Rodríguez L, Lopez-Alonso I, et al. Angiotensin-converting enzymes (ACE, ACE2) gene variants and COVID-19 outcome. Gene. 2020;762:145102.

9. Chiu RWK, Tang NLS, Hui DSC, Chung GTY, Chim SSC, Chan KCA, et al. ACE2 gene polymorphisms do not affect outcome of severe acute respiratory syndrome. ClinChem. 2004;50(9):1683-6.

10. Wei J, Alfajaro M, Hanna R, DeWeirdt P, Strine M, Lu-Culligan W, et al. Genome-wide CRISPR screens reveal host factors critical for SARS-CoV-2 infection. Cell. 2021;184(1):76-91.

Publisher's Note Springer Nature remains neutral with regard to jurisdictional claims in published maps and institutional affiliations. 